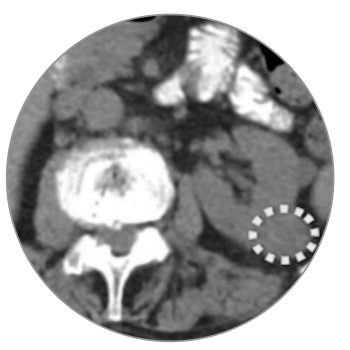

$\gg$

\section{Palabras clave (DeCS)}

Oncocitoma

Neoplasias renales

Diagnóstico

Tomografía

computarizada

multidetector

\section{Key words (MeSH)}

Oncocytoma

Kidney neoplams

Diagnosis

Multidetector computed tomography

\title{
Oncocitomas: hallazgos tomográficos y correlación anatomopatológica
}

\author{
Oncocytomas: Imaging Findings and Anatomopathology \\ Correlation
}

\author{
Jorge Ocantos \\ Martina Aineseder \\ Roy López Grove \\ Diego Santillán² \\ Mario Gueglio²
}

\begin{abstract}
Resumen
Introducción: En imágenes, los hallazgos del oncocitoma generalmente coinciden con el carcinoma de células renales (CCR) por lo que resulta muy poco segura su discriminación mediante imágenes. Objetivos: Evaluar el comportamiento de una muestra de oncocitoma en tomografía (TC). Métodos: Se seleccionaron retrospectivamente los pacientes con cirugía de tumor renal y diagnóstico anatomopatológico de oncocitoma, que fueron tratados entre enero de 2015 y diciembre de 2017. Se incluyeron los pacientes a quienes se les realizó TC con medio de contraste endovenoso en nuestra institución. Resultados: Del total de pacientes $(n=44)$, 43 tenían una lesión única renal, mientras que uno tenía tres lesiones. Del total de las lesiones $(n=47), 20$ (42,55 \%) fueron diagnosticadas tras una nefrectomía radical y 24 (51,10\%) fueron diagnosticadas por una nefrectomía parcial. La media de diámetro máximo fue de 36,5 mm ( $R I Q 22-44,25)$, de los cuales se agruparon en menores y mayores a $4 \mathrm{~cm}$; se encontraron 22 tumores en este último grupo (47\%). De estos, en 15 tumores $(31,91 \%)$ que tenían más de $4 \mathrm{~cm}$ se encontró la cicatriz central. Se evidenciaron calcificaciones en 3 pacientes (6,8 \%). Se encontró 1 tumor (2,1\%) con inversión de realce segmentario luego de la administración medio de contraste endovenoso. En este caso, el oncocitoma era menor a $4 \mathrm{~cm}$. Conclusión: El hallazgo de una masa sólida con realce más intenso que el parénquima circundante durante la fase nefrográfica obliga a considerar al oncocitoma entre los diagnósticos diferenciales
\end{abstract}

\begin{abstract}
Summary
Introduction: The imaging findings of oncocytomas usually coincide with renal cell carcinoma (RCC), which makes it difficult to discriminate them in imaging. Objective: To evaluate the imaging findings of a sample of oncocytomas in tomography (CT). Methods: We retrospectively selected patients with renal tumor surgery and oncocytoma anatomopathological diagnosis, who were treated between January 2015 and December 2017. Patients who underwent CT with intravenous contrast at our institution were included. Results: Of the total number of patients ( $\mathrm{n}$ $=44), 43$ had a single renal lesion while one patient presented 3 lesions. Of the total lesions $(n=$ 47), 20 (42.55\%) were diagnosed after a radical nephrectomy and 24 (51.10\%) were diagnosed by a partial nephrectomy. The mean maximum diameter was $36.5 \mathrm{~mm}$ (RIQ 22-44, 25), of which they were grouped by tumor length into smaller or larger than $4 \mathrm{~cm}$, with 22 tumors in this last group (47\%). Of these, 15 tumors (31.91\%) that were larger than $4 \mathrm{~cm}$ had a central scar. Calcifications were evident in 3 patients (6.8 \%). One tumor (2.1\%) was found with the presence of inversion of segmental enhancement after administration of intravenous contrast. In this case, the oncocytoma was less than $4 \mathrm{~cm}$. Conclusion: The finding of a solid mass with more enhancement than the surrounding parenchyma during the nephrographic phase makes it necessary to consider oncocytoma among the differential diagnoses.
\end{abstract}

\section{Introducción}

El oncocitoma renal es un tumor benigno y representa el 3-7 \% de las neoplasias renales (1).

Los oncocitomas renales se caracterizan por un realce homogéneo hipervascular con lavado en la fase tardía por tomografía computarizada (TC) y un realce heterogéneo en el caso de aquellos mayores a $4 \mathrm{~cm}$ (2), sin hemorragia, calcificación o necrosis (3). En lesiones de gran tamaño es frecuente encontrar una cicatriz central (4)
Los hallazgos por imágenes de los oncocitomas coinciden en general con el carcinoma de células renales (CCR), por lo que este método resulta muy poco seguro para su diferenciación (3).

\section{Métodos}

Se seleccionaron retrospectivamente los pacientes con cirugía de tumor renal y diagnóstico anatomopatológico de oncocitoma, que fueron tratados entre enero de 
2015 a diciembre de 2017. Se incluyeron los pacientes a quienes se les tomaron TC con medio de contraste endovenoso. De 70 pacientes, se excluyeron aquellos que no contaban con el protocolo de cuatro fases para evaluar lesiones renales, con lo cual se obtuvo una muestra de 44 pacientes y 47 lesiones.

A dichos pacientes se les realizaron TC multicorte con tomógrafos de 64 y 320 pistas (Toshiba Aquilion y Aquilion One, Otawara, Japón). Las imágenes se obtuvieron en apnea inspiratoria y con los siguientes parámetros: $120 \mathrm{kVp}$, corriente de tubo variable y un espesor de corte de $1 \mathrm{~mm}$. El protocolo de estudio consta de cuatro fases: una fase sin medio de contraste endovenoso desde las bases pulmonares hasta las crestas iliacas, inyección de 100-150 mL medio de contraste iodado no iónico con un flujo de $3 \mathrm{~mL} / \mathrm{s}$. Luego de la administración de medio de contraste, se obtuvieron una fase corticomedular (40-45 segundos), nefrográfica (90-120 segundos) y una excretora (7-10 minutos).

Para el análisis de las imágenes se clasificaron las fases en: fase sin medio de contraste, cuando no se había administrado contraste endovenoso; corticomedular, si el realce de la corteza predominaba sobre el de la médula (pirámides); nefrográfica, cuando todo el parénquima renal es homogéneo y excretora, cuando el medio de contraste se encontraba opacificando el sistema pielocalicial. Las imágenes fueron analizadas e interpretadas en una estación de trabajo (DICOM Medical Alma 5.0). Las TC fueron revisadas por un especialista en imágenes genitourinarias con 11 años de experiencia, que solo tenía información de la edad y el género de los pacientes.

Para el análisis se emplearon dos ROI de entre $0,5-1 \mathrm{~cm}^{2}$ en el área de mayor realce del medio de contraste en el caso de las lesiones heterogéneas y en el centro de las lesiones con realce homogéneo y se calcularon las medias de realce. Se compararon estos realces con la densidad del parénquima sano adyacente en cada una de las fases (figura 1). El diámetro máximo de cada lesión fue medida en cortes axiales.

De los 44 pacientes se analizaron las lesiones y se evaluaron según tamaño, patrón de realce, magnitud de realce, calcificaciones, cicatriz central y signo de la inversión de realce segmentario; es decir, sectores de la lesión que se mostraban hiperdensos e hipodensos en la fase corticomedular, se comportan en forma contraria en la fase nefrográfica. Estos hallazgos se evaluaron bajo un sistema de organización de variables dicotómicas.

El análisis estadístico fue calculado usando el software SPSS (SPSS 18 para Windows, SPSS, Chicago).

\section{Resultados}

Del total de pacientes $(n=44), 43$ presentaban una lesión única renal, mientras que un paciente presentaba 3 lesiones (figura 2). Del total de las lesiones $(n=47), 20(42,55 \%)$ fueron diagnosticadas tras una nefrectomía radical y $24(51,10 \%)$ fueron diagnosticadas por una nefrectomía parcial.

Del total, 27 pacientes eran mujeres $(61,4 \%)$. La media de edad al momento de la nefrectomía fue de 70 años (RIQ 62,5 a 76,5). En cuanto a las características tumorales, 43 eran sólidos $(91,1 \%), 3$ sólido-quísticos $(6,4 \%$ ) y uno quístico (2,2 \%) (figura3).

La media de diámetro máximo fue de 36,5 mm (RIQ 22-44,25), de los cuales se agruparon en menores (figura 4 ) y mayores a $4 \mathrm{~cm}$, habiendo 22 tumores en este último grupo (47\%).
La cicatriz central fue encontrada en 15 tumores $(31,91 \%)$, de los cuales todos presentaban un tumor mayor a $4 \mathrm{~cm}$ (figura 5). Se evidenciaron calcificaciones en 3 pacientes $(6,8 \%)$.

Se encontró un tumor $(2,1 \%)$ con inversión de realce segmentario luego de la administración de contraste endovenoso (figura 6). En este caso, el oncocitoma era menor a $4 \mathrm{~cm}$ (tabla 1 ).

Tabla 1. Características de los tumores en TC

\begin{tabular}{|c|c|}
\hline Muestra & $\boldsymbol{n}=\mathbf{4 7}$ \\
\hline Mayores a $4 \mathrm{~cm}$ & $22(47 \%)$ \\
\hline Cicatriz central & $15(31,91 \%)$ \\
\hline Calcificaciones & $3(6,3 \%)$ \\
\hline Inversión de realce segmentario & $1(2,1 \%)$ \\
\hline
\end{tabular}

La media de densidad tumoral en las fases sin medio de contraste, corticomedular, nefrográfica y excretora fueron de 26,8 UH (r 25,727,9), 150,7 UH (r 146,6-151,8), 155,6 UH (r 150,6-160,6) y 93,7 UH (r 89,8-97,6), con mayor pico de realce en la fase nefrográfica.

La relación entre la densidad del tumor y el parénquima normal adyacente no fue significativa en la fase sin medio de contraste $(1 \pm$ $2,4, p 0,42)$. Se encontró diferencia significativa entre la densidad del parénquima normal y la fase corticomedular $(25,6 \pm 11,6, p 0,0001)$, la nefrográfica $(43 \pm 10, p 0,0001)$ y la fase excretora $(27,3 \pm 5, p$ 0,0001) (figura 7).

\section{Discusión}

Numerosas publicaciones han informado que aproximadamente un $20 \%$ de las masas renales menores a $4 \mathrm{~cm}$ y entre un 5 y un $10 \%$ de las mayores a $4 \mathrm{~cm}$ demuestran ser lesiones benignas luego de la cirugía (5-7).

Además, teniendo en cuenta que la nefrectomía, tanto radical como parcial, es innecesaria en este tipo de lesiones, numerosos autores han intentado caracterizarlas para diferenciarlas de las malignas.

Varios autores, en diferentes publicaciones, han intentado identificar características distintivas de los oncocitomas teniendo en cuenta que representan la segunda lesión benigna renal más frecuente. Entre las características tomográficas de los oncocitomas renales se incluyen márgenes definidos, realce homogéneo, cicatriz central y la inversión segmentaria de realce.

El realce con medio de contraste se define con el aumento mayor a $10 \mathrm{UH}$ entre la imagen sin y con medio de contraste, aunque otros autores plantean que el realce entre 10-20 UH es indeterminado (8). El pseudorrealce de las masas renales pequeñas puede ocurrir como resultado del aumento en la densidad del parénquima renal normal circundante y debe considerarse especialmente con las masas endofíticas (9).

El grado de realce tumoral y la fase de mayor realce es variable, según lo comunicado en diferentes publicaciones. En el estudio de Bird y colaboradores (10) se demostró que el oncocitoma muestra mayor realce en la fase corticomedular mientras que en otros estudios, como el de Pierorazio y colaboradores o el de Zhang y colaboradores, se observó en la fase nefrográfica $(11,12)$. La variabilidad reportada en el realce con contraste coincide con nuestros resultados. 

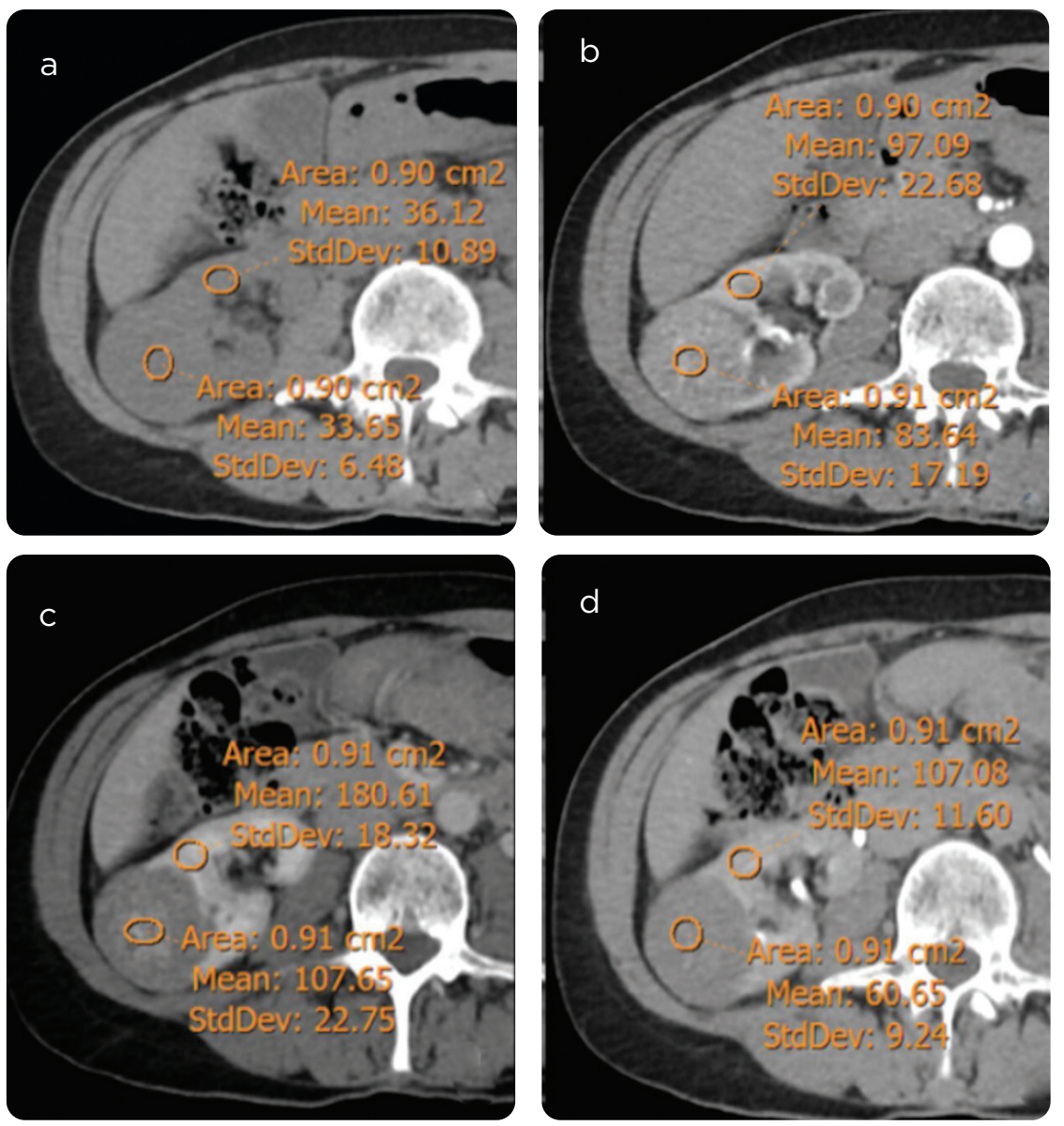

Figura 1. TC multifásica en una mujer de 70 años. a) TC, fase sin medio de contraste, b) corticomedular, c) nefrográfica y d) excretora. Formación heterogénea en el polo superior del riñón izquierdo. La densidad de la lesión y del parénquima adyacente \pm SD fue calculada en cada fase.
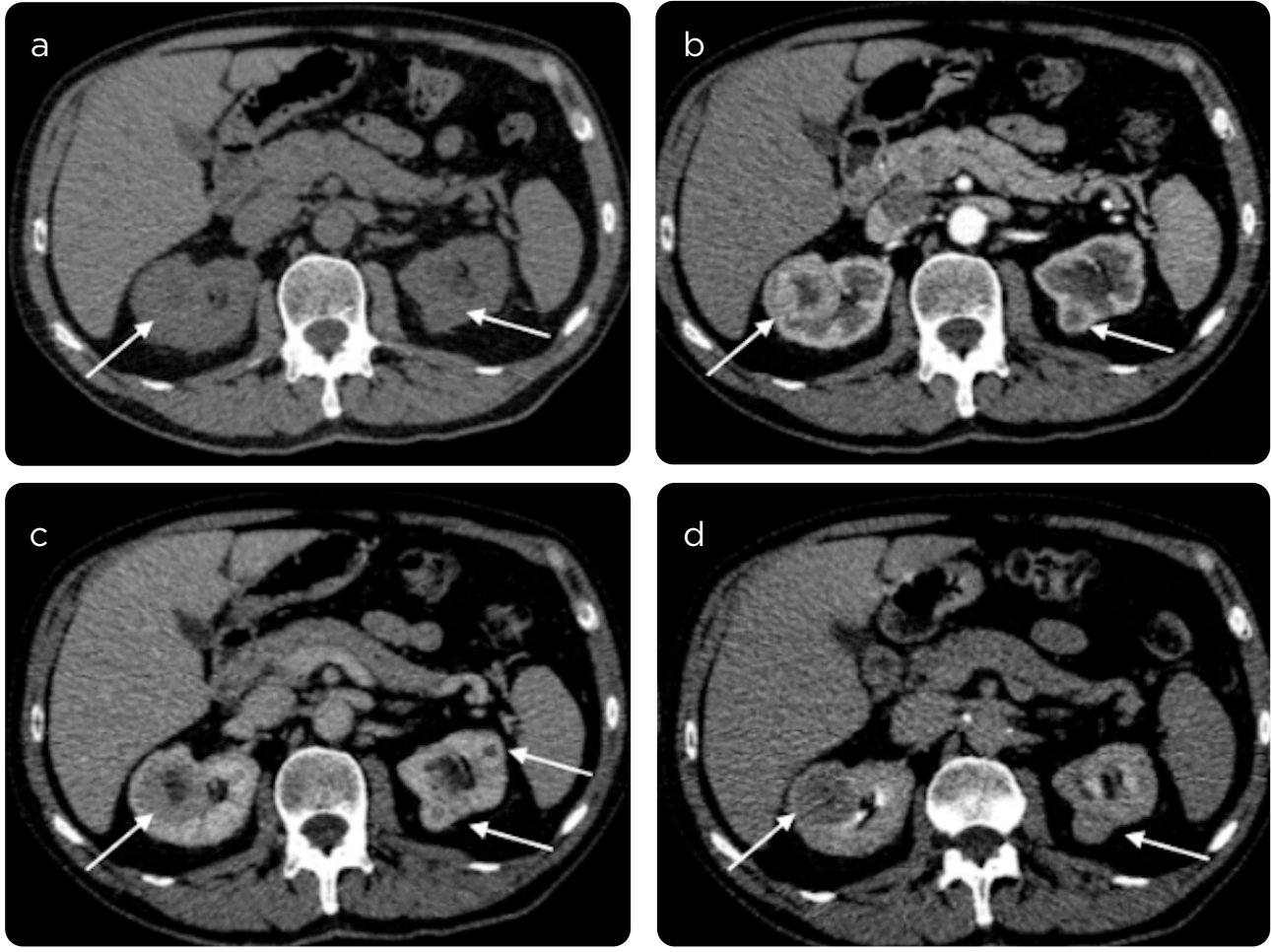

Figura 2. Oncocitomas bilaterales. a) TC, fase sin medio de contraste, b) corticomedular, c) nefrográfica y d) excretora, en ambos riñones, con realce periférico temprano, mientras que el sector central de los tumores tiene menor realce, en relación con la cicatriz central. Los tumores muestran menor realce relativo respecto al parénquima renal sano, el área central no puede diferenciarse de necrosis que podría presentar un carcinoma de células renales. 

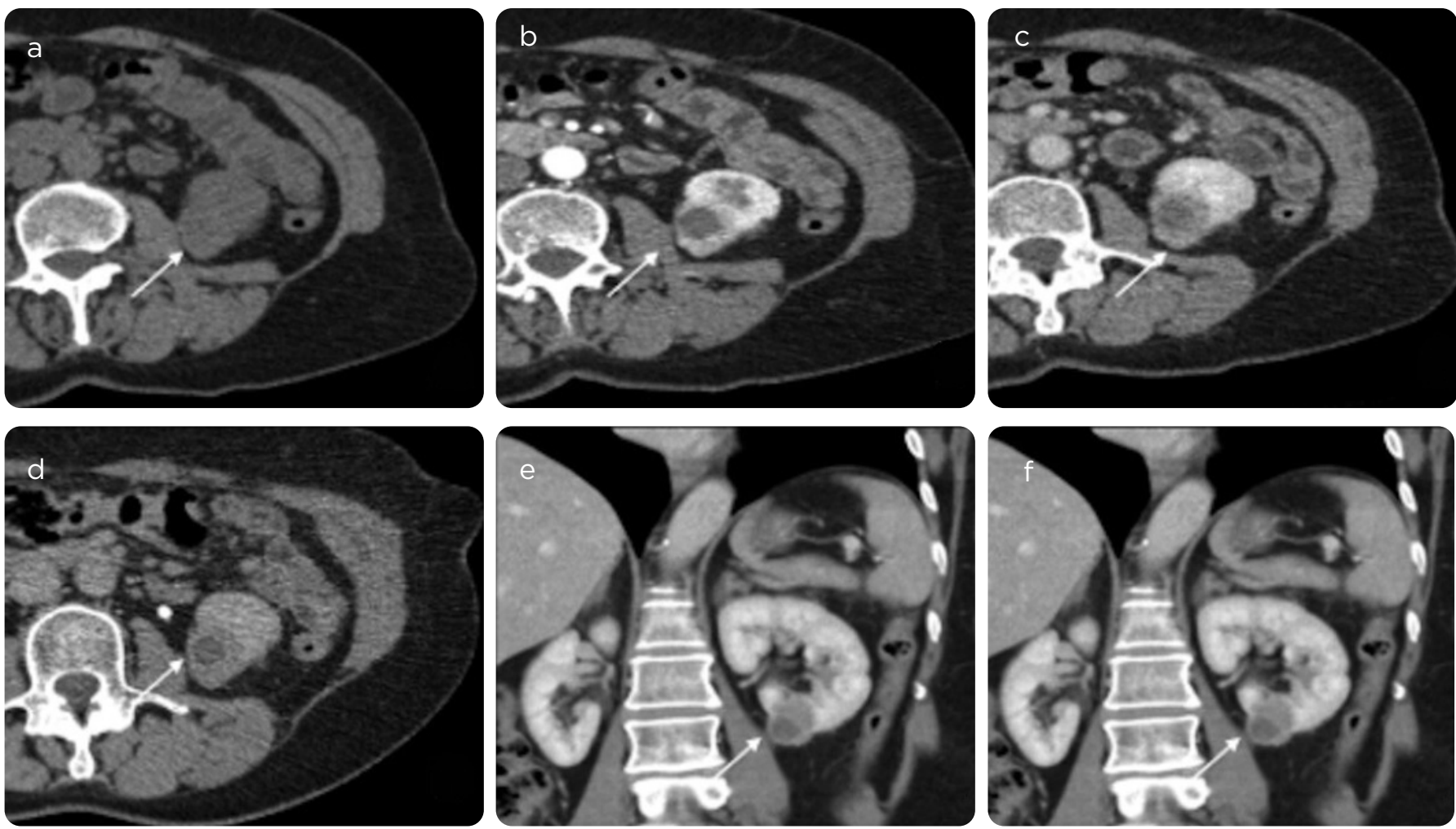

Figura 3. Oncocitoma sólido-quístico. TC, a) fase sin medio de contraste, corticomedular (b axial, e coronal y f sagital), c) nefrográfica axial y d) excretora axial. Formación sólido-quística en polo inferior de riñón izquierdo. Región central de baja densidad que no realza en las fases con medio de contraste y el resto del tumor realza sobretodo en la fase corticomedular (b), aunque en menor medida en relación con el parénquima adyacente. La zona sin realce no puede diferenciarse de necrosis que podría encontrarse en carcinoma renal con necrosis o variedades quísticas.

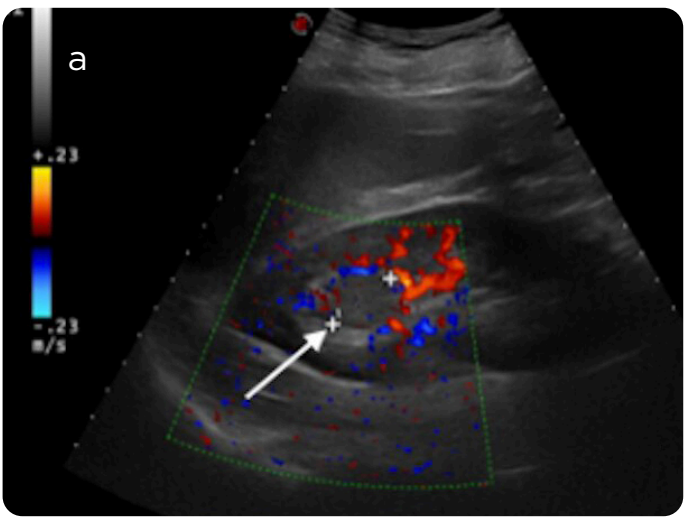

C

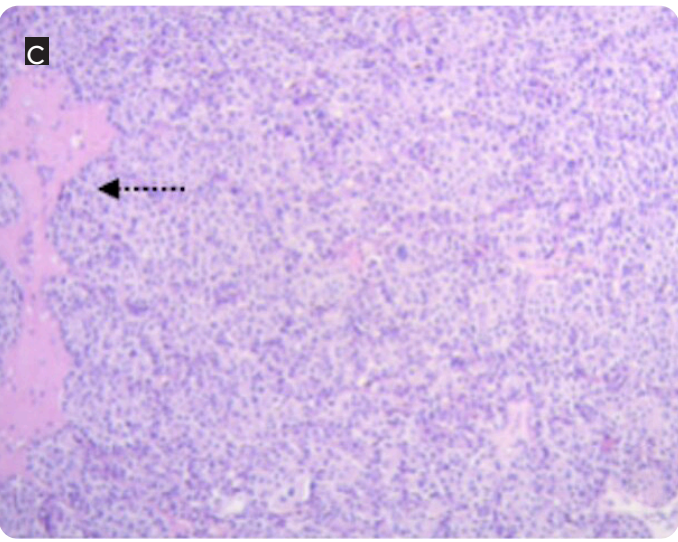

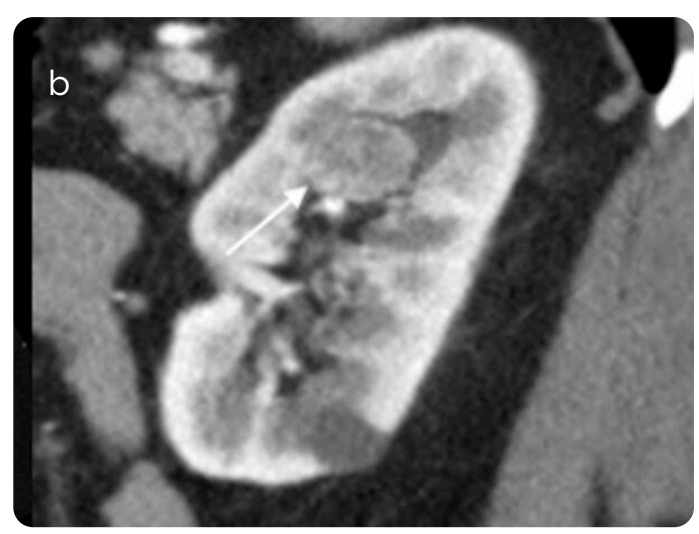

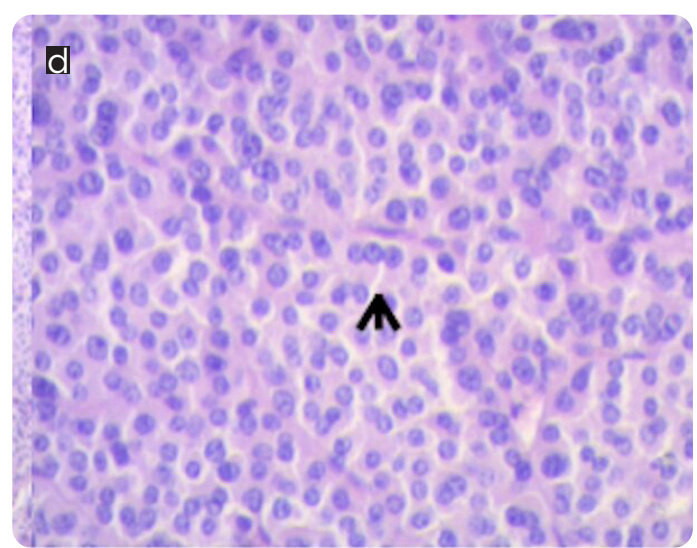

Figura 4. Correlación entre ecografía y TC. a) Ecografía renal y TC en fase corticomedular (b) reconstrucción sagital) Formación nodular menor a 4 $\mathrm{cm}$ que se insinúa en el seno renal (flecha), que presenta flujo Doppler en su interior y periférico a). En TC se evidencia la formación (flecha) que desplaza levemente el grupo calicial superior y presenta realce heterogéneo luego de la administración de contraste endovenoso. c) Histología con hematoxilina y eosina $100 x$ $y$ d) 400x, que evidencia un patrón sólido con estructuras vasculares (flecha punteada negra) y células oncocíticas con núcleos con atipia degenerativa (punta de flecha negra). 

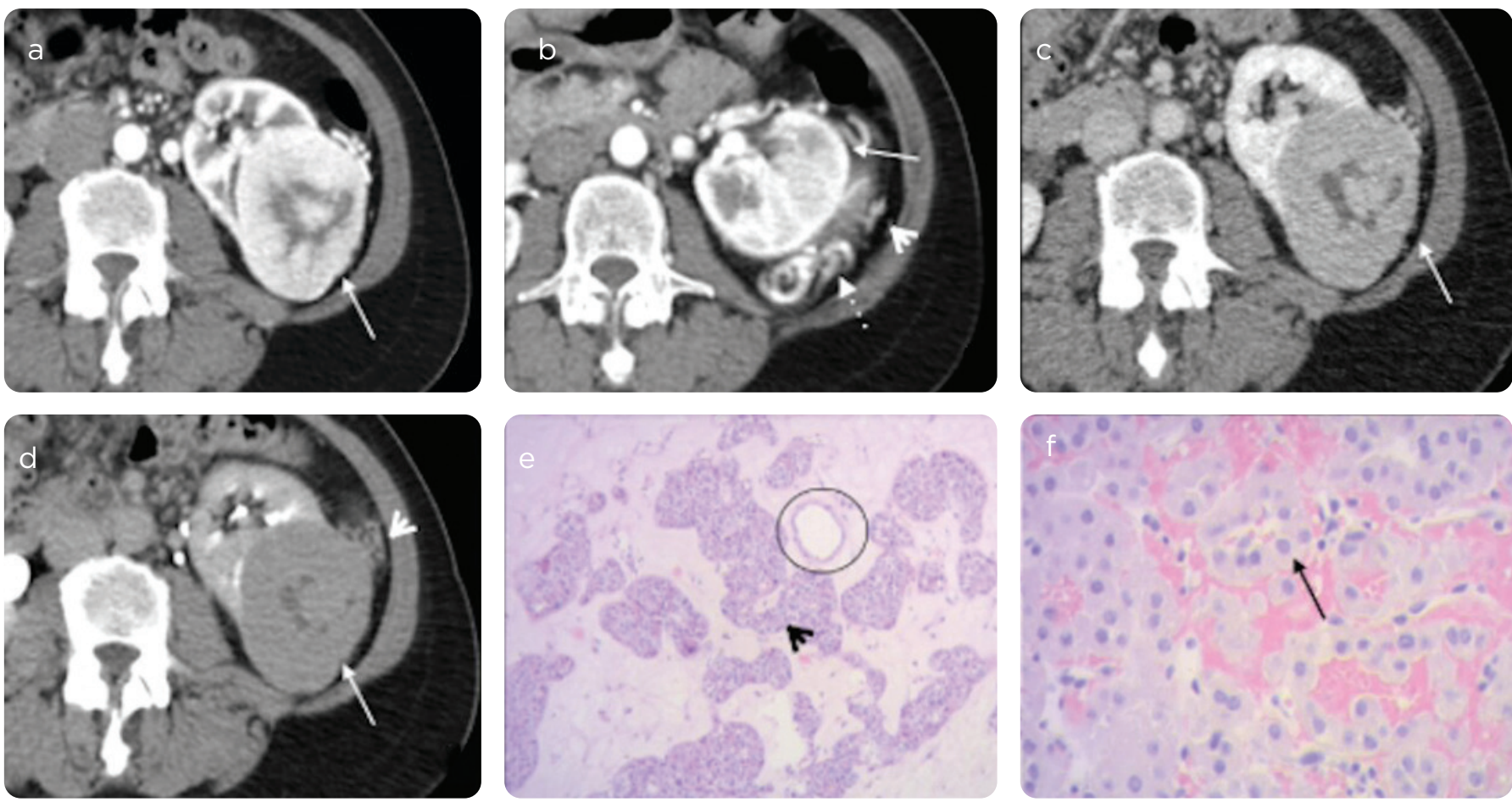

Figura 5. Oncocitoma con cicatriz central. TC, a y b) fase corticomedular, c) nefrográfica y d) excretora. Formación sólida (flecha), con dos segmentos, uno de alta densidad periférico y otro de baja densidad central en fase corticomedular. Neovascularización perirrenal (flecha punteada). Aumento en la densidad de los planos grasos adyacentes (cabeza de flecha). El comportamiento de la zona central visible en este caso no permite diferenciarlo de necrosis o isquemia. e) Histología con hematoxilina y eosina 100x y f) 400x en donde se evidencian áreas edematosas con nidos de células (cabeza de flecha negra) con vasos delicados (círculo) y células oncocíticas con núcleos redondeados y citoplasma eosinófilo (flecha negra).
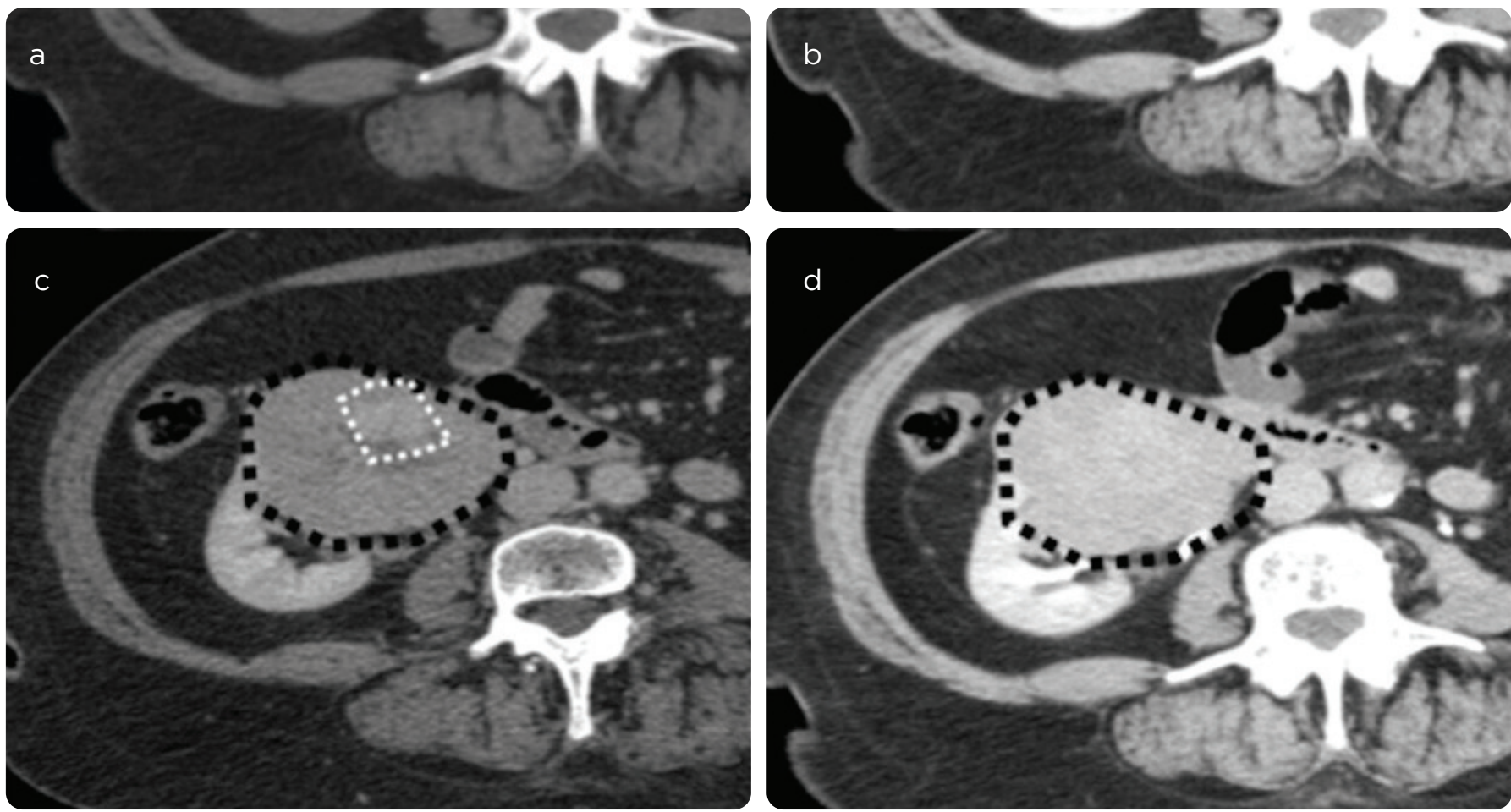

Figura 6. Inversión segmentaria de realce. TC, a) fase sin contraste, b) corticomedular, c) nefrográfica y d) excretora. c) muestra realce del tumor (línea punteada negra), excepto por la región central (línea punteada blanca). d) muestra inversión del realce segmentario de la masa. El segmento central en esta fase es de alta densidad con respecto a la periferia, a la inversa de lo observado en la fase corticomedular. En la fase de excretora se pierden las diferencias entre ambos componentes tumorales. 

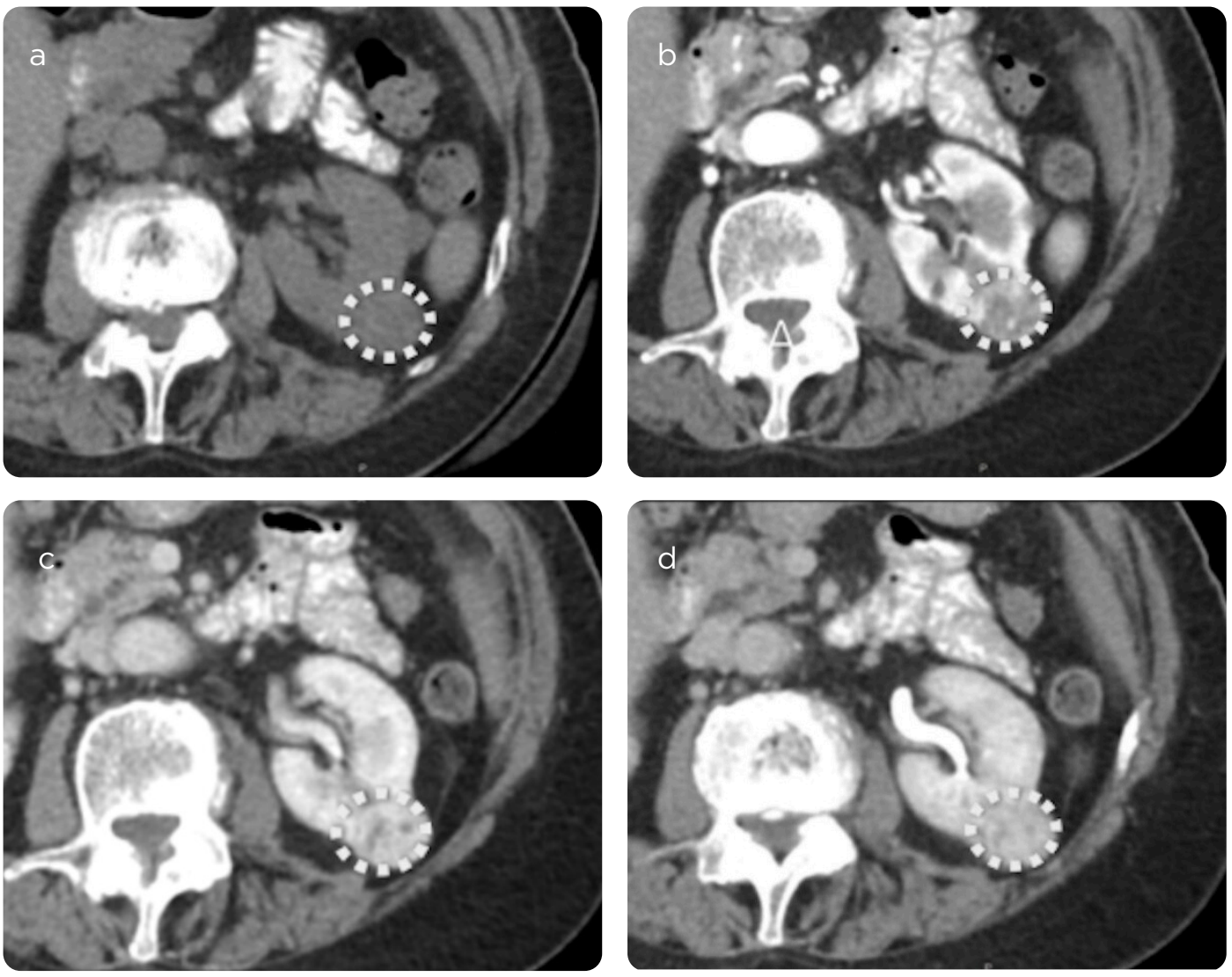

Figura 7. Oncocitoma sólido, pequeño, con realce heterogéneo. TC, a) fase sin medio de contraste, b) corticomedular, c) nefrográfica y d) excretora. Tumor menor a $4 \mathrm{~cm}$ (línea punteada). La fase corticomedular (b) evidencia un área focal central de baja densidad en la lesión. La fase nefrográfica pone de manifiesto un área focal de baja densidad en la misma región.

Kim y colaboradores (13) describieron un patrón segmentario con inversión en las fases corticomedular y nefrográfica en los oncocitomas de pequeño tamaño. El oncocitoma tiene dos componentes, evidenciables luego de la administración del medio de contraste, en la fase corticomedular, uno de alta y el otro de baja atenuación, pero en la fase nefrográfica los patrones se invierten.

Sin embargo, algunos estudios encontraron que este patrón de realce es controversial para los oncocitomas (14-16). En esta serie de casos solo se presentó un caso con inversión del realce. Un motivo en la discrepancia de la prevalencia de este hallazgo es atribuible a las diferencias en los protocolos de TC utilizados (1). Aunque en este estudio se utilizó un protocolo estandarizado y se excluyeron aquellos pacientes que no hubieran sido estudiados en cuatro fases tomográficas, se debe tener en cuenta que este hallazgo aparece en un $6 \%$ de los oncocitomas y en aquellos oncocitomas menores a $4 \mathrm{~cm}$ (14). Otra explicación posible es que la histoarquitectura de los oncocitomas es más variable de lo que se piensa.

La heterogeneidad subjetiva tumoral, luego de la administración del medio de contraste endovenoso, es un importante parámetro en la caracterización tumoral $(3,12,16)$. Los oncocitomas tienden a ser más homogéneos que los CCR (17).

La cicatriz central corresponde a una zona central de tejido conectivo, con bandas fibróticas proyectadas hacia la periferia de la lesión. Se ha informado en el $33 \%$ de los casos, y es más frecuente encontrarla en lesiones mayores a $25 \mathrm{~mm}$ (1). Sin embargo, no es un signo patognomónico y es indiferenciable de la necrosis central que suelen presentar los CCR (18). En esta muestra, dicho signo se vio en 15 tumores $(31,25 \%)$ y en su totalidad fueron lesiones mayores a $4 \mathrm{~cm}$.
Diferenciar una masa benigna de una maligna representa el principal objetivo en la caracterización de las lesiones renales, lo que contribuye a reducir la incidencia de procedimientos innecesarios (7), aunque es difícil diferenciar los oncocitomas de las lesiones malignas como el CCR cromófobo (19).

Teniendo en cuenta las dificultades que las imágenes suelen presentar en la diferenciación certera de una lesión benigna de una maligna, la biopsia de masa renales de pequeño tamaño puede resultar de utilidad cuando se plantea la posibilidad de realizar una cirugía mayor (20) o procedimientos mínimamente invasivos, como ablación por radiofrecuencia o crioablación (21).

La radiómica se define como la conversión de imágenes a datos cuantitativos, y la subsiguiente extracción de estos datos para un mejor soporte de decisiones (22). Las características cuantitativas de la imagen basadas en la intensidad, la forma, el tamaño o el volumen y la textura, ofrecen información sobre el fenotipo tumoral y el microentorno (o hábitat), son diferentes de lo que proporcionan los resultados de las pruebas de laboratorio y los análisis genómicos y proteómicos o los informes imagenológicos convencionales (22). El análisis de estos biomarcadores de imagen podría ayudar en la caracterización de oncocitomas, sin necesidad de someter al paciente a una cirugía innecesaria (22).

El carácter retrospectivo de este trabajo representa una debilidad. En esta primera etapa de análisis se realizó un estudio descriptivo de las variables mencionadas, por lo que se valoró la frecuencia de aparición de las mismas, la comparación con otras poblaciones no forma parte de esta presentación, y constituye un análisis aún en progreso para futuras comparaciones. 
En todos los casos estudiados en este grupo hubo confirmación quirúrgica anatomopatológica y son escasos los trabajos con este número de población de muestra, aún menor en habla hispana (de acuerdo con la búsqueda bibliográfica existen dos artículos). Por último, todos los tumores se estudiaron con el mismo protocolo, con el fin de eliminar la variabilidad de la técnica.

El realce heterogéneo con un pico en la fase nefrográfica fue el hallazgo que mejor caracterizó en este estudio a los oncocitomas, tanto mayores como menores de $4 \mathrm{~cm}$.

En el caso de los menores de $4 \mathrm{~cm}$, este hallazgo combinado con la inversión de realce segmentaria fueron los que se encontraron con mayor frecuencia, y los que combinados ayudan a identificar estas lesiones.

En cuanto a los mayores de $4 \mathrm{~cm}$, el realce heterogéneo en fase nefrográfica junto con la presencia de una cicatriz central de baja densidad, fueron los hallazgos combinados más frecuentes.

\section{Conclusión}

El hallazgo de una masa sólida con realce más intenso que el parénquima circundante durante la fase nefrográfica obliga a considerar al oncocitoma entre los diagnósticos diferenciales. Cuando se trata de lesiones menores a $4 \mathrm{~cm}$, la inversión segmentaria refuerza dicha posibilidad.

\section{Referencias}

1. Woo S, Cho JY, Kim SH, Kim SY, Lee HJ, Hwang SI, et al. Segmental enhancement inversion of small renal oncocytoma: differences in prevalence according to tumor size. AJR Am J Roentgenol. 2013;200(5):1054-9.

2. Gakis G, Kramer U, Schilling D, Kruck S, Stenzl A, Schlemmer H-P. Small renal oncocytomas: Differentiation with multiphase CT. Eur J Radiol. 2011;80:274-8. http:/ dx.doi.org/10.1016/j.ejrad.2010.06.049

3. Davidson AJ, Hayes WS, Hartman DS, McCarthy WF, Davis CJ. Renal oncocytoma and carcinoma: failure of differentiation with CT. Radiology. 1993;186:693-6. http:// dx.doi.org/10.1148/radiology.186.3.8430176

4. Chao DH, Zisman A, Pantuck AJ, Freedland SJ, Said JW, Belldegrun AS. Changing concepts in the management of renal oncocytoma. Urology. 2002;59(5):635-42.

5. Duchene DA, Lotan Y, Cadeddu JA, Sagalowsky AI, Koeneman KS. Histopathology of surgically managed renal tumors: analysis of a contemporary series. Urology. 2003;62:827-30. http://dx.doi.org/10.1016/s0090-4295(03)00658-7

6. Filipas D, Fichtner J, Spix C, Black P, Carus W, Hohenfellner R, et al. Nephronsparing surgery of renal cell carcinoma with a normal opposite kidney: long-term outcome in 180 patients. Urology. 2000;56:387-92. http://dx.doi.org/10.1016/s00904295(00)00656-7

7. Frank I, Blute ML, Cheville JC, Lohse CM, Weaver AL, Zincke H. Solid renal tumors: an analysis of pathological features related to tumor size. J Urol. 2003;170(6 Pt 1):2217-20.

8. Israel GM, Bosniak MA. How I Do It: Evaluating renal masses. Radiology. 2005;236:441-50. http://dx.doi.org/10.1148/radiol.2362040218

9. Stakhovskyi O, Yap SA, Leveridge M, Lawrentschuk N, Jewett MAS. Small renal mass: what the urologist needs to know for treatment planning and assessment of treatment results. AJR Am J Roentgenol. 2011;196(6):1267-73.

10. Bird VG, Kanagarajah P, Morillo G, Caruso DJ, Ayyathurai R, Leveillee R, et al. Differentiation of oncocytoma and renal cell carcinoma in small renal masses $(<4$ $\mathrm{cm})$ : the role of 4-phase computerized tomography. World J Urol. 2011;29(6):787-92.

11. Pierorazio PM, Hyams ES, Tsai S, Feng Z, Trock BJ, Mullins JK, et al. Multiphasic enhancement patterns of small renal masses $(\leq 4 \mathrm{~cm})$ on preoperative computed tomography: utility for distinguishing subtypes of renal cell carcinoma, angiomyolipoma, and oncocytoma. Urology. 2013;81(6):1265-71.

12. Zhang J, Lefkowitz RA, Ishill NM, Wang L, Moskowitz CS, Russo P, et al. Solid renal cortical tumors: differentiation with CT. Radiology. 2007;244(2):494-504.

13. Kim JI, Cho JY, Moon KC, Lee HJ, Kim SH. Segmental enhancement inversion at biphasic multidetector CT: characteristic finding of small renal oncocytoma. Radiology. 2009;252(2):441-8

14. McGahan JP, Lamba R, Fisher J, Starshak P, Ramsamooj R, Fitzgerald E, et al. Is Segmental Enhancement Inversion on Enhanced Biphasic MDCT a reliable sign for the noninvasive diagnosis of renal oncocytomas? Am J Roentgenol. 2011;197:W674-9. http://dx.doi.org/10.2214/ajr.11.6463

15. Schieda N, McInnes MDF, Cao L. Diagnostic accuracy of segmental enhancement inversion for diagnosis of renal oncocytoma at biphasic contrast enhanced CT: systematic review. Eur Radiol. 2014;24(6):1421-9.
16. O'Malley ME, Tran P, Hanbidge A, Rogalla P. Small renal oncocytomas: is segmental enhancement inversion a characteristic finding at biphasic MDCT? AJR Am J Roentgenol. 2012;199(6):1312-5.

17. Sasaguri K, Takahashi N, Gómez-Cardona D, Leng S, Schmit GD, Carter RE, et al Small $(<4 \mathrm{~cm})$ Renal Mass: Differentiation of Oncocytoma from renal cell carcinoma on biphasic contrast-enhanced CT. AJR Am J Roentgenol. 2015;205(5):999-1007.

18. Galia M, Albano D, Bruno A, Agrusa A, Romano G, Di Buono G, et al. Imaging features of solid renal masses. Br J Radiol. 2017;90(1077):20170077.

19. Kuroda N, Toi M, Yamamoto M, Miyazaki E, Hayashi Y, Hiroi M, et al. Immunohistochemical identification of intracytoplasmic lumens by cytokeratin typing may differentiate renal oncocytomas from chromophobe renal cell carcinomas. Histo Histopathol. 2004;19(1):23-8.

20. Beland MD, Mayo-Smith WW, Dupuy DE, Cronan JJ, DeLellis RA. Diagnostic yield of 58 consecutive imaging-guided biopsies of solid renal masses: should we biopsy all that are indeterminate? AJR Am J Roentgenol. 2007;188(3):792-7.

21. Heilbrun ME, Zagoria RJ, Julian Garvin A, Craig Hall M, Krehbiel K, Southwick A, et al. CT-Guided biopsy for the diagnosis of renal tumors before treatment with percutaneous ablation. Am J Roentgenol. 2007;188:1500-5. http://dx.doi.org/10.2214/ ajr.06.0389

22. Gillies RJ, Kinahan PE, Hricak H. Radiomics: Images are more than pictures, they are data. Radiology. 2016;278(2):563-77.

\section{Correspondencia}

Martina Aineseder

Perón 4190, Buenos Aires, Argentina

martina.aineseder@hospitalitaliano.org.ar

Recibido para evaluación: 10 de noviembre de 2019 Aceptado para publicación: 20 de febrero de 2020 